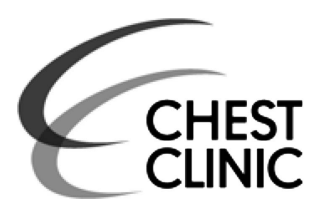

Department of Medicine, Toronto General Hospital, University of Toronto, Ontario, Canada

\section{Correspondence to}

Dr John Thenganatt, Division of Respirology, Department of Medicine, University Health Network, Toronto General Hospital, 585 University Avenue, 9N 965, Toronto, ON, Canada M5G 2N2; john. thenganatt@uhn.ca

Received 22 August 2013 Accepted 9 October 2013 Published Online First 25 October 2013

\title{
Connect the dots: a rare case of spontaneous haemopneumothorax
}

\author{
Natalie K Kozij, John Thenganatt
}

A 19-year-old woman presented to her local emergency department with acute chest pain and shortness of breath. She denied any cough, haemoptysis, fever or leg swelling.

Her past medical history was significant for premature birth at 28 weeks gestational age. She required surgical correction of a right clubfoot as an infant. In addition, she had intestinal malrotation complicated by caecal volvulus and perforation treated with laparotomy at age 13 , and an ovarian cystectomy performed at age 17 . There was no family history of pneumothorax or respiratory disease. She was a non-smoker on no medications.

On examination, she was tachycardic, tachypnoeic and had an oxygen saturation in the low 80 s. Her respiratory exam demonstrated decreased air entry to bilateral lung bases. A chest radiograph demonstrated bilateral pneumothoraces and chest tubes were inserted emergently. A CT scan of the chest demonstrated cystic lucencies in the left lung with areas of consolidation (figure 1). Laboratory investigations were unremarkable.

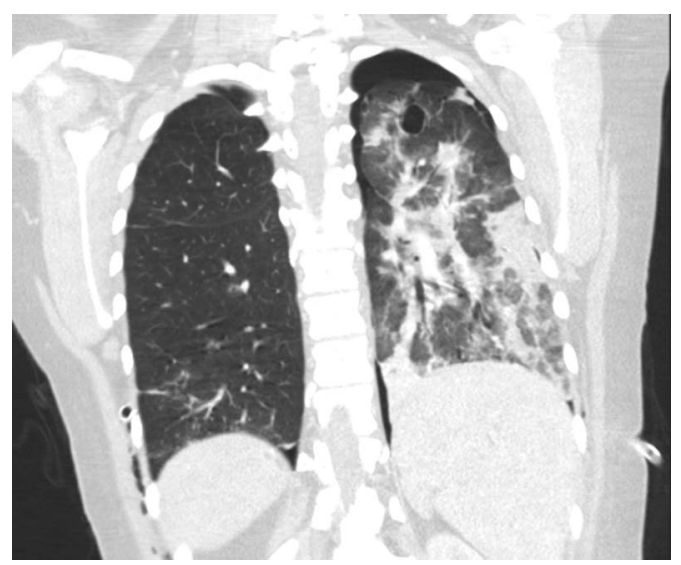

Figure 1 Coronal image from an unenhanced CT scan of the chest, post chest tube insertion. Note the evidence of persistent bilateral pneumothoraces, more notable on the left. There is extensive left-sided consolidation and a cystic lesion in the left upper lobe. (a)

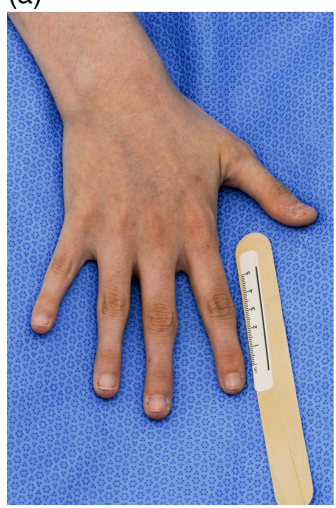

(b)

Figure 2 (A) Photo of the patient's right hand, demonstrating tapering fingers characteristic of EhlersDanlos syndrome. (B) Photo of the patient's left knee, demonstrating hypopigmented lesions with thinning of the skin known as 'cigarette paper' scars (both photos taken by PhotoGraphics Studio, University Health Network).

Her left-sided pneumothorax did not resolve with chest tube insertion, so thoracoscopic pleurodesis was performed along with a left upper lobe wedge biopsy. Pathology revealed widespread intra-alveolar haemorrhage and hemosiderin-laden macrophages. In some sections, blood appeared to be filling an emphysematous bulla, surrounded by organising fibrosis and chronic inflammation.

On further examination, she was noted to have hyperextensibility of the elbows, wrists and metacarpophalangeal joints with tapering fingers (figure $2 \mathrm{~A}$ ). Her skin was translucent with dilated blood vessels. She was noted to have hypopigmented lesions over her knees consistent with 'cigarette paper' scars (figure 2B).

For Answer see page 301

Contributors NKK: literature review, summary of case, generation of manuscript. JT: staff supervisor/editor.

Competing interests None.

Provenance and peer review Not commissioned; externally peer reviewed. 


\section{Connect the dots: a rare case of spontaneous haemopneumothorax}

\section{ANSWER}

From the question on page 298

\section{WHAT IS THE DIAGNOSIS?}

Given her constellation of findings, she was referred to a medical geneticist who confirmed a diagnosis of Ehlers-Danlos syndrome (EDS) Type IV with a pathogenic mutation in the COL3A1 gene.

EDS is a heterogeneous group of disorders secondary to abnormalities in Type III collagen. EDS Type IV is the most severe form, manifesting with vascular fragility leading to arterial rupture, bowel perforation and uterine rupture. In a retrospective review of 419 individuals with EDS Type IV, ${ }^{1}$ there was an increased incidence of prematurity, congenital clubfoot and congenital dislocation of the hip. Life expectancy is estimated at 48 years, and $25 \%$ of affected individuals have their first manifestation before the age of 20 years. The most common cause of death is related to arterial rupture.

Pneumothorax is the most common pulmonary complication due to tears in the lung parenchyma resulting in pulmonary haemorrhage. ${ }^{1}$ Structural lung abnormalities that develop include thick-walled cavities from previous parenchymal rupture, bullous lung disease, panacinar emphysema, pulmonary cysts and bronchiectasis. ${ }^{2} 3$

In our patient, MRI of the vascular tree showed no aneurysmal dilatations. She was assessed by an obstetrician and advised to use contraception, given the high risk of uterine rupture associated with this condition.

EDS Type IV is an uncommon but important clinical entity with multiple systemic effects. In the absence of an alternative diagnosis, individuals should be examined for clinical features suggestive of collagen diseases when presenting with spontaneous haemothorax or pneumothorax.

\section{REFERENCES}

1 Pepin M, Schwarze U, Superti-Furga A, et al. Clinical and genetic features of EhlersDanlos syndrome type IV, the vascular type. N Engl J Med 2000;342:673-80. [Erratum, N Engl J Med 2001;344:392.]

2 Selim B, Lane CR, Rubinowitz AN, et al. Spontaneous Hemothorax and Recurrent Hemoptysis in a 26-Year-Old Man With Skin Lesions. Chest 2010;137:480-3.

3 Dowton SB, Pincott S, Demmer L. Respiratory complications of Ehlers-Danlos syndrome type IV. Clin Genet 1996;50:510-14. 